\title{
METRIZATIONS OF PROJECTIVE SPACES
}

\author{
HERBERT BUSEMANN
}

A two-dimensional $G$-space, ${ }^{1}$ in which the geodesic through two distinct points is unique, is either homeomorphic to the plane $E^{2}$ and all geodesics are isometric to a straight line, or it is homeomorphic to the projective plane $P^{2}$ and all geodesics are isometric to the same circle, see $[1, \S \S 10$ and 31$]$.

Two problems arise in either case: (1) To determine the systems of curves (in $E^{2}$ or $P^{2}$ ) which occur as geodesics. (2) If the geodesics are (or lie on) ordinary straight lines, can the space be imbedded in a higher-dimensional space with the ordinary straight lines as geodesics? The author solved both these problems for $E^{2},[1$, Theorems (11.2) and (14.8)], but left both open for $P^{2}[1$, Appendix (9) and (10)]. Recently Skornyakov [2] solved the first problem for $P^{2}$; he modified the author's basic idea through replacing a summation by an integration, and thus eliminated the singularities which the author's procedure would produce in the case of $P^{2}$.

The purpose of this note is to show that a device similar to Skornyakov's can be used to solve Problem (2) for $P^{n}$. Our method also provides a much simpler solution of Problem (1). Thus we are going to prove simultaneously:

Theorem I. Let the projective space $P^{n}, n \geqq 2$, be metrized as a $G$ space such that the geodesics are the projective lines. Then $P^{n}$ can be imbedded in $P^{n+1}$ (and hence in $P^{m}$ with $m>n$ ) such that the metric in $P^{n}$ is preserved and the geodesics in $P^{n+1}$ are the projective lines.

Theorem II (of Skornyakov). In $P^{2}$ let a system $\Sigma^{\prime}$ of curves be given such that each curve in $\Sigma^{\prime}$ is a closed Jordan curve and two distinct points of $P^{2}$ lie on exactly one curve in $\Sigma^{\prime}$. Then $P^{2}$ can be metrized as a G-space such that the curves in $\Sigma^{\prime}$ become the geodesics.

For the proof we pass to the sphere $S^{n}$ or $S^{2}$ as universal covering space of $P^{n}$ or $P^{2}$. An $r$-dimensional spherical sub-space of $S^{n}$ with the maximal radius will be denoted as a "great $S^{r}$." We refer to the hypotheses of the two theorems as Cases I and II respectively. In Case I there is one great $S^{n}$ in $S^{n+1}$, which we denote by $Q$ and which

Received by the editors April 21, 1956.

1 Except for differentiability hypotheses, a $G$-space is a complete symmetric Finsler space. For the exact definition see $\$ 8$ in [1]. The results of [1] will be freely used. 
is already metrized such that the geodesics are the great circles in $Q$; we have to extend the metric from $Q$ to $S^{n+1}$.

In Case II we obtain from $\Sigma^{\prime}$ a system $\Sigma$ of curves on $S^{2}$ which are closed Jordan curves and have the property of containing with any point of $S^{2}$ also its antipodal point, because a curve in $\Sigma^{\prime}$ does not decompose $P^{2}$. We select any curve $Q$ in $\Sigma$ and two antipodal points $w$, $w^{\prime}$ not on $Q$. By a topological mapping of $S^{2}$ on itself we can reach that $Q$ and the curves in $\Sigma$ through $w$ and $w^{\prime}$ become ordinary great circles. This normalization implies that with the ordinary twodimensional measure on $S^{2}$ all curves in $\Sigma$ have measure 0 , because a curve in $\Sigma$ not through $w$, $w^{\prime}$ is a closed set and intersects each curve through $w$ and $w^{\prime}$ exactly twice.

A semi-circle $K_{p}$ is an arc from $p$ to its antipodal point $p^{\prime}$ on an ordinary great circle in Case I and on a curve in $\Sigma$ in Case II. For $x \neq p, p^{\prime}$ there is exactly one $K_{p}$ which passes through $x$ and which we denote by $K_{p}(x)$.

$Q$ decomposes $S^{n}, n \geqq 2$, into two open hemispheres $H$ and $H^{\prime}$. For an arbitrary point $p \in H$, its antipode $p^{\prime} \in H^{\prime}$ and $x \neq p, p^{\prime}$ we put $x_{p}=K_{p}(x) \cap Q$. The mapping $x \rightarrow x_{p}$ is continuous for $x \neq p, p^{\prime}$. (For the continuity properties of $\Sigma$ compare the analogous arguments for $E^{2}$ in $[1$, pp. 57,58$\left.]\right)$. Also:

(1) $x \rightarrow x_{p}$ maps antipodes in $S^{n+1}$ on antipodes in $Q$ and $x_{p}=x$ for $x \in Q$. In Case I a great circle not through $p$ is mapped by $x \rightarrow x_{p}$ on a great circle in $Q$.

In Case I the metric in $Q$ is given. In Case II we introduce on $Q$ the spherical distance or any other distance that makes $Q$ isometric to a circle such that antipodes in the sense of $Q$ coincide with antipodes on $S^{2}$. Let $2 \lambda$ be the common length (see $[1,(31.2)]$ ) of the geodesics in $Q$ in Case I or of $Q$ in Case II. We put

$$
f_{p}(x, y)=x_{p} y_{p} \quad \text { for } x \neq p, p^{\prime}, y \neq p, p^{\prime} .
$$

Then

(3) $f_{p}(x, y)=f_{p}(y, x) \geqq 0$ with equality only for $K_{p}(x)=K_{p}(y)$,

(4) $f_{p}(x, y)+f_{p}(y, z) \geqq f_{p}(x, z)$;

here the equality holds only if $K_{p}(x)=K_{p}(y)$ or $K_{p}(y)=K_{p}(z)$ or if $x_{p}, y_{p}, z_{p}$ lie on a semicircle in $Q$ and $y_{p}$ between $x_{p}$ and $z_{p}$.

(5) With $f_{p}(x, y)$ as distance any semicircle which contains neither $p$ nor $p^{\prime}$ is isometric to a segment of length $\lambda$, i.e., to the interval $0 \leqq t \leqq \lambda$ with $\left|t_{1}-t_{2}\right|$ as distance.

$$
f_{p}(x, y)=x y
$$

for $x, y \in Q$. 
The assertions (5) and (6) follow from (1) and (2). Finally we put

$$
f_{p}(p, x)=f_{p}(x, p)=f_{p}\left(p^{\prime}, x\right)=f_{p}\left(x, p^{\prime}\right)=0 .
$$

Then $f_{p}(x, y) \leqq \lambda$ for all $p, x, y$. For fixed $x, y$ the function $f_{p}(x, y)$ is continuous in $p$ for $p \neq x, x^{\prime}, y, y^{\prime}$ and is lower semi-continuous at the latter points. Therefore, if we use on $H$ a measure proportional to the ordinary spherical measure so normalized that $\int_{H} d p=1$, then

$$
\rho(x, y)=\int_{H} f_{p}(x, y) d p
$$

will exist as a Riemann integral. Because of (6)

$$
\rho(y, x)=x y \quad \text { for } x, y \in Q .
$$

(9) Any semicircle is with $\rho(x, y)$ as distance isometric to a segment of length $\lambda$, hence any great circle (or curve in $\Sigma$ ) is isometric to a circle of length $2 \lambda$.

Let $G$ be the great circle containing the given semicircle $K$. If $x, y \in K$ then

$$
\int_{H} f_{p}(x, y) d p=\int_{H-G} f_{p}(x, y) d p .
$$

On the other hand, if $p_{i} \notin G, a_{i}>0, \Sigma a_{i}=1, i=1,2, \cdots, m$, then (5) implies that $K$ is with the distance $\Sigma a_{i} f_{p_{i}}(x, y)$ a segment of length $\lambda$. Since $\int_{H-G f_{p}}(x, y) d p$ can be interpreted as Riemann integral, (9) follows.

$$
\begin{gathered}
\rho(x, y)=\rho(y, x) \geqq 0 \text { with equality only for } x=y \text { and } \\
\rho(x, y)+\rho(y, z) \geqq \rho(x, y)
\end{gathered}
$$

follow from (3), (9) and (4). Finally:

$$
\rho(x, y)+\rho(y, z)>\rho(x, z) \text { if } x, y, z \text { do not lie on one great circle. }
$$

For Case I the proof is immediate: For any $p$ not in the great $S^{2}$ determined by $x, y, z$ the points $x_{p}, y_{p}, z_{p}$ do not lie on one great circle in $Q$, hence $x_{p} y_{p}+y_{p} z_{p}>x_{p} z_{p}$. Since $f_{p}(x, y)=x_{p} y_{p}$ is a continuous function of $p$, when $p$. is not in the great $S^{2}$, the assertion follows from (4) and (7).

In both cases, no two of the points $x, y, z$ can be antipodal because then a great circle containing all three points would exist. Hence $x_{y}$ and $z_{y}$ are by (1) not antipodal in $Q$ and we can find a semicircle in $Q$ with end points $q, q^{\prime}$ say, which contains $x_{y}$ and $z_{y}$ as interior 
points. If $p$ lies on $K_{y}(q)$ close to $y$ then $y_{p}=q$ or $y_{p}=q^{\prime}$ and $x_{p}, z_{p}$ lie close to $x_{y}$ and $z_{y}$, consequently there is a semicircle with $y_{p}$ as one end point which contains both $x_{p}$ and $z_{p}$, so that $x_{p} y_{p}+y_{p} z_{p}>x_{p} z_{p}$.

Since it is clear from the continuity properties of $f_{p}(x, y)$ that the distance $\rho(x, y)$ is topologically equivalent to the spherical distance on $S^{n+1}$ or $S^{2}$, our theorems follow from (8), (9) and (11) after identifying antipodal points, see [1, pp. 128, 129].

The reasons for the brevity of the present proof of Theorem II as compared to Skornyakov's are: using a double instead of single integral and, principally, metrizing $S^{2}$ instead of a euclidean plane obtained from $P^{2}$ by cutting it along a curve $C$ in $P^{2}$ (traversed twice). Showing that the metric satisfies Theorem II after reidentification of diametrically opposite points on $C$ is the main difficulty in [2]. This raises the question whether the proofs of theorems $[1$, (14.8)] corresponding to Theorem I for $E^{n}$ and $[1,(11.2)]$ corresponding to Theorem II for $E^{2}$ can be similarly simplified and unified. Topological peculiarities, like asymmetry of the asymptote relation, see $[1,(23.5)]$, seem to indicate that some complications are unavoidable.

\section{REFERENCES}

1. H. Busemann, The geometry of geodesics, New York, 1955.

2. L. A. Skornyakov, Metrization of the projective plane in connection with a given system of curves, (Russian), Izvestiya Akad. Nauk SSSR, Ser. Mat. vol. 19 (1955) pp. $471-482$.

University of Southern California 